\title{
Changing microbiology of indwelling venous catheter related infection
}

\author{
Lokesh Shahani ${ }^{*}$, Chad Noggle ${ }^{2}$ and Nancy Khardori ${ }^{3}$ \\ ${ }^{1}$ McGovern Medical School, The University of Texas Health Science Center at Houston, Houston, TX, USA \\ ${ }^{2}$ Southern Illinois University School of Medicine, Springfield, IL, USA \\ ${ }^{3}$ Eastern Virginia Medical School, Norfolk, VA, USA
}

\begin{abstract}
Background: Indwelling venous catheters (IVCs) have become an integral part of managing patients in both inpatient and outpatient settings. Even with advances in technology and techniques, infectious complications remain highly prevalent and result in significant morbidity and mortality. Various patient and catheter related factors have been previously studied to be associated with IVC related infections.

Aim: The purpose of this study was to investigate the correlation between the microbiology of IVC related infections and various factors such as patient co morbidities, type of catheter used and the indication for placement.

Methods: We retrospectively studied patients admitted to the university-affiliated hospital for IVC related infections from October 2008 to December 2010.

Results: Our data show an increase in the incidence of Staphylococcus aureus infections (28\%) as compared to the previously reported national data. Staphylococcus aureus was associated with various risk factors, such as catheter use for antibiotic administration $(\mathrm{OR}=6.2)$, $\mathrm{PICC}$ line insertion $(\mathrm{OR}=2.378)$, diabetes mellitus $(\mathrm{OR}=1.43)$, hemodialysis $(\mathrm{OR}=1.411)$, and tunneled catheter use $(\mathrm{OR}=1.4)$. Individuals with catheters placed for hemodialysis were 2.35 times more likely to have infections with coagulase negative Staphylococci, and 1.397 times more likely to present with other gram positive organism. Similarly, individuals receiving TPN were 1.188 times more likely to have Candida species as the infecting organism.
\end{abstract}

Conclusion: These correlations are important for choosing initial presumptive antimicrobial therapy, duration of antimicrobial therapy and pursuing catheter removal.

Abbreviations: Indwelling venous catheter-IVC; Coagulase negative Staphylococci-CONS; Peripherally inserted central catheter-PICC; Total parenteral nutrition-TPN

\section{Introduction}

Indwelling central venous catheters (IVCs) have become an integral and essential to the management of diverse illnesses in and out of the hospital. They provide secure access to the central circulation for infusion therapy, nutritional support, hemodynamic monitoring, plasmapheresis and hemodialysis. IVC related infections have substantially decreased in the ICU setting in the past decade; however there has been an increasing trend noted in other inpatient and dialysis unit settings. The total number of IVC related blood stream infections estimated to occur each year in United States ICUs, dialysis units, and inpatient units is 78,000 [1].

SCOPE (Surveillance and Control of Pathogens of Epidemiological Importance), a nationwide surveillance study of nosocomial bloodstream infections in the U.S estimated a significant variation in the microbiology of the IVC related infections [2]. Coagulase negative Staphylococci (CONS) accounted for the majority i.e. $31 \%$ of the infections followed by gram negative bacilli (22\%), Staphylococcus aureus (20\%), Enterococcus spp. (9\%) and Candida spp. (9\%). Similarly according to the "US National Nosocomial Infections Surveillance System Report", the pathogens most frequently associated with IVC infections from 1991-1999 were: CONS (37\%), Staphylococcus aureus (13\%), Enterococcus spp. (13\%), and Candida albicans (8\%) (3). Both these large studies found CONS to be the predominant organism in the 1990s. Smaller hospital based studies have shown a rise in infections caused by gram-negative organism and Staphylococcus aureus, however there is variation in these results.

Various patient and catheter related factors have been studied as variables that affect the rate of catheter related infections. The primary goal of our study was to generate the microbiology data for IVC related infections for our affiliated hospital. Further, the aim of this study was to investigate the correlation between the microbiology of IVC infections and various factors such as patient co morbidities, type of catheter used and the indication for placement. The results would have implications in choosing initial presumptive antimicrobial therapy and the decision to remove the indwelling vascular access.

\section{Methods}

A total of 204 participants were included in the current study by way of a retrospective chart review conducted on patients admitted

${ }^{\star}$ Correspondence to: Lokesh Shahani, McGovern Medical School, The University of Texas Health Science Center at Houston, TX,191 East Road, Houston, TX 77054, USA, Tel: 217-801-1985; E-mail: Lokesh.R.Shahani@uth.tmc.edu

Key words: indwelling venous catheter related infection, microbiology, Staphylococcus aureus

Received: June 01, 2018; Accepted: June 28, 2018; Published: July 02, 2018 
to the university-affiliated hospital due to IVC related infection from October 2008 to December 2010. The institutional review board approved the study.

The hospital course of the patients was reviewed for information on demographic characteristics such as age and gender. Potential risk factors at the time of diagnosis of IVC related infection were examined such as length of hospital stay, indications for catheter placement, type of catheter and presence of co morbidities. The host risk factors or the co morbidities extracted were diabetes mellitus, end stage renal disease, HIV infection, hematological malignancy and administration of chemotherapy and steroids. Indwelling venous catheters included peripherally inserted central catheter (PICC), tunneled IVCs, nontunneled IVCs and implanted catheters.

SPSS was used to calculate descriptive statistics, relative risks, and determine the level of significance by way of using the Continuity Correction Chi-Square. Odds ratios were then calculated by hand. Three specific analyses were run on the data. This included evaluations of the (1) risk of IVC infection based on catheter type, (2) risk of IVC infection based on the indication for catheter placement, and (3) risk of IVC infection based on patient comorbities.

\section{Results}

\section{Descriptive Statistics}

A total of 204 hospitalizations with IVC related infections were found. The types of IVC were PICC (114 or 58\%), tunneled IVCs (45 or $23 \%$ ), non - tunneled IVCs (18 or 9\%) and implanted catheters (20 or $10 \%)$. The age range of patients in our study was 18 to 92 years (median $=58$ years). Males and females were equally distributed (51.7\% vs. $49.3 \%$ ). Ninety-three percent were Caucasian, 5\% African American, and the remaining $2 \%$ Hispanic, Asian American, Native American, or classified as other. The average length of hospital stay was 17.58 days (range $1-98$ days). IVC related infections were caused by CONS (33\%), Staphylococcus aureus (28\%), gram negative bacilli (18\%), other gram positive organisms (14\%) and Candida spp. (8\%).

\section{IVC infection and catheter type}

Using Chi-square, significant associations were made between PICC and the occurrence of Staphylococcus aureus $\left(\chi^{2}=6.920\right.$, $\mathrm{p}=0.009)$. Additionally, use of a tunneled catheter was associated with a significant increased risk of Staphylococcus aureus $\left(\chi^{2}=6.582, p=0.01\right)$. Results suggested individuals with a PICC were 2.378 times more likely to present with Staphylococcus aureus and individuals with a tunneled catheter were 1.4 times more likely to present with Staphylococcus aureus as compared to patients with other types of catheters (Table 1).

In comparison, no associations were made between the use of PICC and the occurrence of CONS $\left(\chi^{2}=3.664, p=0.056\right)$, gram negative bacilli $\left(\chi^{2}=0.256, p=0.613\right)$, other gram positive organisms $\left(\chi^{2}=1.623\right.$, $\mathrm{p}=0.203)$, or Candida spp. $\left(\chi^{2}=1.137, \mathrm{p}=0.286\right)$. Use of a tunneled catheter was not linked to an increased risk of CONS $\left(\chi^{2}=2.264\right.$, $\mathrm{p}=0.132)$, gram negative bacilli $\left(\chi^{2}=0.629, \mathrm{p}=0.428\right)$ or other gram positive organism $\left(\chi^{2}=3.331, \mathrm{p}=0.068\right)$. Non - tunneled catheters were not associated with a higher risk of any of the examined organisms. Specifically, use of non - tunneled catheters was not associated with an increased risk of Staphylococcus aureus $\left(\chi^{2}=0.060, p=0.806\right)$, CONS $\left(\chi^{2}\right.$ $=0.013, p=0.910)$, gram negative bacilli $\left(\chi^{2}=0.342, p=0.559\right)$, other gram positive organisms $\left(\chi^{2}=0.021, p=0.884\right)$, or Candida spp. $\left(\chi^{2}=0.072\right.$, $\mathrm{p}=0.789$ ).
Finally, no associations were made between the use of implanted catheters and the increased risk of infection with any of the examined organisms. Specifically, use of implanted catheters was not associated with an increased risk of Staphylococcus aureus $\left(\chi^{2}=0.251, p=0.617\right)$, CONS $\left(\chi^{2}=0.864, p=0.353\right)$, gram negative bacilli $\left(\chi^{2}=0.080, p=0.778\right)$, other gram positive organisms $(\chi=0.099, p=0.753)$, or Candida spp. $\left(\chi^{2}=2.101, \mathrm{p}=0.147\right)$.

\section{IVC infection and indication for catheter placement}

Significant associations were found between hemodialysis catheters and the occurrence of Staphylococcus aureus $\left(\chi^{2}=6.359, \mathrm{p}=0.012\right)$, CONS $\left(\chi^{2}=4.549, \mathrm{p}=0.033\right)$, and other gram positive organisms $\left(\chi^{2}=4.276\right.$, $\mathrm{p}=0.039$ ). In comparison, hemodialysis was not associated with an increased risk of gram negative bacilli $\left(\chi^{2}=0.579, p=0.448\right)$ or Candida spp. $\left(\chi^{2}=2.381, \mathrm{p}=0.121\right)$. Antibiotic administration was associated with a significant increased risk of Staphylococcus aureus $\left(\chi^{2}=7.453\right.$, $\mathrm{p}=0.006)$. However, antibiotic administration was not associated with an increased risk of CONS $\left(\chi^{2}=1.711, \mathrm{p}=0.191\right)$, gram negative bacilli $\left(\chi^{2}=2.614, p=0.103\right)$ Candida spp. $\left(\chi^{2}=0.026, p=0.872\right)$, or other gram positive organisms $\left(\chi^{2}=0.003, p=0.959\right)$. Total parenteral nutrition (TPN) administration was associated with an increased risk of Candida spp. $\left(\chi^{2}=8.749, \mathrm{p}=0.002\right)$, but not an increased risk of Staphylococcus aureus $\left(\chi^{2}=0.225, \mathrm{p}=0.636\right)$, CONS $\left(\chi^{2}=0.007, \mathrm{p}=0.933\right)$, gram negative bacilli $\left(\chi^{2}=0.479, p=0.489\right)$, or other gram positive organisms $\left(\chi^{2}=0.325\right.$, $\mathrm{p}=0.569)$. Finally, chemotherapy administration was not associated with an increased risk of Staphylococcus aureus $\left(\chi^{2}=0.402, p=0.526\right)$, CONS $\left(\chi^{2}=0.001, p=0.974\right)$, gram negative bacilli $\left(\chi^{2}=0.159, p=0.690\right)$, Candida spp. $\left(\chi^{2}=.451, \mathrm{p}=.502\right)$, or other gram positive organisms $\left(\chi^{2}=0.017, \mathrm{p}=0.897\right)$ (Table 2).

In regards to relative risk, individuals presenting with hemodialysis are 1.411 times more likely to present with Staphylococcus aureus, 2.35 times more likely to present with CONS, and 1.397 times more likely to present with another gram positive organism as compared to individuals not on hemodialysis. Individuals undergoing antibiotic administration are 6.2 times more likely to present with and Staphylococcus aureus as compared to individuals not on antibiotics. Finally, individuals with TPN administration are 1.188 times more likely to present with Candida species as compared to individuals not on TPN.

\section{IVC infection and patient co-morbidities}

The third and final analysis involved an evaluation of the risks of IVC related infections based on patient comorbidities. Across all comparisons, only one significant association was found between the presence of IVC infection and diabetes mellitus. Specifically, a significant association was found between history of diabetes mellitus and the occurrence of Staphylococcus aureus $(\chi 2=2.743, \mathrm{p}=0.049)$. Results suggested individuals with a history of diabetes mellitus were 1.432 times more likely to present with Staphylococcus aureus as compared to non - diabetics. No significant association was found between diabetes mellitus and other organisms. Furthermore, no significant associations were found between the other assessed co-morbidities and various organisms associated with IVC related infections (Table 3).

\section{Discussion}

We report an increase in the incidence of IVC related infections due to Staphylococcus aureus (28\%) as compared to the previously reported national data. Staphylococcus aureus was associated with various risk factors, such as catheter use for antibiotic administration $(\mathrm{OR}=6.2)$, PICC line insertion $(\mathrm{OR}=2.378)$, diabetes mellitus $(\mathrm{OR}=1.43)$, 
Table 1. Correlation between IVC type and microbiology of IVC related infections.

\begin{tabular}{|c|c|c|c|c|}
\hline Catheter type & Organisms & $\chi^{2}$ & p value & OR \\
\hline PICC line & Staphylococcus aureus & 6.92 & 0.009 & 2.378 \\
\hline PICC line & CONS & 3.664 & 0.056 & 0.533 \\
\hline PICC line & Gram negative bacilli & 0.256 & 0.613 & 0.822 \\
\hline PICC line & Other gram positive organism & 1.623 & 0.203 & 1.739 \\
\hline PICC line & Candida species & 1.137 & 0.286 & 0.527 \\
\hline Tunneled catheter & Staphylococcus aureus & 6.582 & 0.01 & 1.4 \\
\hline Tunneled catheter & CONS & 2.264 & 0.132 & 1.864 \\
\hline Tunneled catheter & Gram negative bacilli & 0.629 & 0.428 & 1.468 \\
\hline Tunneled catheter & Other gram positive organism & 3.331 & 0.068 & 0.438 \\
\hline Non - tunneled catheters & Staphylococcus aureus & 0.06 & 0.806 & 0.873 \\
\hline Non - tunneled catheters & CONS & 0.013 & 0.910 & 1.065 \\
\hline Non - tunneled catheters & Gram negative bacilli & 0.342 & 0.559 & 0.705 \\
\hline Non - tunneled catheters & Other gram positive organism & 0.021 & 0.884 & 1.121 \\
\hline Non - tunneled catheters & Candida species & 0.072 & 0.789 & 1.331 \\
\hline Implanted catheters & Staphylococcus aureus & 0.251 & 0.617 & 0.722 \\
\hline Implanted catheters & CONS & 0.864 & 0.353 & 1.71 \\
\hline Implanted catheters & Gram negative bacilli & 0.08 & 0.778 & 1.203 \\
\hline Implanted catheters & Other gram positive organism & 0.099 & 0.753 & 1.277 \\
\hline Implanted catheters & Candida species & 2.101 & 0.147 & 0.376 \\
\hline
\end{tabular}

Table 2. Correlation between indication of IVC placement and microbiology of IVC related infections.

\begin{tabular}{|c|c|c|c|c|}
\hline Indication & Organisms & $\chi^{2}$ & p value & OR \\
\hline Hemodialysis & Staphylococcus aureus & 6.359 & 0.012 & 1.411 \\
\hline Hemodialysis & CONS & 4.549 & 0.033 & 2.35 \\
\hline Hemodialysis & Gram negative bacilli & 0.579 & 0.448 & 1.418 \\
\hline Hemodialysis & Other gram positive organism & 4.276 & 0.039 & 1.397 \\
\hline Hemodialysis & Candida species & 2.381 & 0.121 & 4.455 \\
\hline Antibiotic administration & Staphylococcus aureus & 7.453 & 0.006 & 6.2 \\
\hline Antibiotic administration & CONS & 1.711 & 0.191 & 0.597 \\
\hline Antibiotic administration & Gram negative bacilli & 2.614 & 0.103 & 0.49 \\
\hline Antibiotic administration & Other gram positive organism & 0.003 & 0.959 & 0.97 \\
\hline Antibiotic administration & Candida species & 0.026 & 0.872 & 1.137 \\
\hline Chemotherapy administration & Staphylococcus aureus & 0.402 & 0.526 & 0.748 \\
\hline Chemotherapy administration & CONS & 0.001 & 0.974 & 1.015 \\
\hline Chemotherapy administration & Gram negative bacilli & 0.159 & 0.69 & 1.259 \\
\hline Chemotherapy administration & Other gram positive organism & 0.017 & 0.897 & 1.088 \\
\hline Chemotherapy administration & Candida species & 0.451 & 0.502 & 2.013 \\
\hline TPN administration & Staphylococcus aureus & 0.225 & 0.636 & 1.287 \\
\hline TPN administration & CONS & 0.007 & 0.933 & 1.041 \\
\hline TPN administration & Gram negative bacilli & 0.479 & 0.489 & 1.561 \\
\hline TPN administration & Other gram positive organism & 0.325 & 0.569 & 1.55 \\
\hline TPN administration & Candida species & 8.749 & 0.002 & 1.188 \\
\hline
\end{tabular}

Table 3. Correlation between diabetes mellitus and microbiology of IVC related infection.

\begin{tabular}{|c|c|c|c|}
\hline Organisms & $\chi^{\mathbf{2}}$ & p value \\
\hline Staphylococcus aureus & $\mathbf{2 . 7 4 3}$ & $\mathbf{0 . 0 4 9}$ & 0.17 \\
\hline CONS & 0.911 & 0.224 & 0.812 \\
\hline Gram negative bacilli & 0.576 & 0.478 & 0.1 \\
\hline Other gram positive organisms & 0.003 & 1.016 \\
\hline
\end{tabular}

hemodialysis $(\mathrm{OR}=1.411)$, and tunneled catheter use $(\mathrm{OR}=1.4)$. Change in the dominant organism in IVC related infections from CONS to Staphylococcus aureus is a highlight of the study. Staphylococcus aureus bacteremia because of the associated complications such as infective endocarditis, osteomyelitis and the risk of re-infection has been associated with a higher morbidity and mortality. Taking into account these factors, the 2009 Infectious Disease Society of America guidelines recommend removal of the catheter if the suspected organism is Staphylococcus aureus along with 4-6 weeks of antimicrobial therapy $[3,4]$. In contrast, CONS, the previously dominant organism in indwelling catheter related infections warrants 1- 2 weeks of antimicrobial therapy with the option of retaining the catheter if an antibiotic lock therapy is used [4].

With the use of indwelling venous catheters in longterm intravenous therapy, the scope of infections has increased. Use of PICC for antibiotic administration is a common example, and both these factors were associated with higher likelihood of presenting 
with Staphylococcus aureus in IVC related infections. The intrinsic adherence factor of the organism plays an important role in the pathogenesis of catheter related infection. As the catheter is in direct contact with the blood stream, the surface becomes coated with platelets and other tissue proteins such as fibrinogen, fibronectin, and laminin [5]. Staphylococcus aureus has the ability to bind to the extracellular matrix proteins [5] and the process of bacterial attachment followed by the multilayered accumulation and intracellular adhesions leads to the formation of biofilms that provide a media for further multiplication of the organisms. Formation of biofilms is a well-studied mechanism in the pathogenesis of IVC related infections, further leading to lack of response and development of resistance to anti-microbial agents.

Further, history of diabetes mellitus increases the risk of Staphylococcus aureus related IVC infections but not the other organisms. Our results show that individuals with a history of diabetes mellitus are 1.432 times more likely to present with Staphylococcus aureus infection compared to non-diabetics. Previously diabetes mellitus was shown to be a risk factor for developing IVC infections when studied in patients who were also on hemodialysis [6]. Staphylococcus aureus infections occur with greater frequency among these patients [7]. Appreciably higher nasal carriage rates of Staphylococcus aureus have been reported among patient with type - II diabetes mellitus [8]. Staphylococcus aureus associated IVC infection is much more common in nasal carriers with three fold higher risk of developing such infection observed in the nasal carrier group [9]. Anterior nares offer an environment conductive for Staphylococcus aureus to colonize and survive for prolonged periods. Phage typing has demonstrated that up to $82.2 \%$ isolates of Staphylococcus aureus recovered from infected vascular accesses and peripheral blood cultures, represent the same strain as the one that colonizes patient's nares [10]. Staphylococcus aureus disseminates from nasal reservoir to hands and skin thus infecting the vascular access sites.

Few studies have sought a link between indications for placement and variations in microbiology of IVC related infections. Isolated studies have indicated predisposition to some bacteria based on patient risk factors. Gram-positive organisms are responsible for most dialysis catheter-related infections. CONS and Staphylococcus aureus together account for 40 to 80 percent of cases in most studies [11-14]. Our study replicated these findings showing that individuals with catheter placed for hemodialysis were 2.35 times more likely to present with CONS, 1.411 times more likely to present with Staphylococcus aureus, and 1.397 times more likely to present with another gram positive organism as compared to individuals not on hemodialysis. Candida blood stream infections are commonly seen among immunosuppressed hosts [15] and occasional outbreaks of Candida infection due to intrinsically contaminated total parenteral nutrition solution have been reported $[16,17]$. Administration of TPN is an important risk factor for candida blood stream infections. Although the mechanism by which TPN increases the risk of candidemia is not well understood, one in vitro study suggested that the lipid emulsion present in TPN solutions increases biofilm production on silicone-elastomer catheters, and supports growth of Candida albicans [18]. In our study individuals on TPN were 1.188 times more likely to present with Candida species as compared to individuals not requiring TPN.

Catheter type was demonstrated to be an important risk factor in a previous study [19]. Factors increasing the risk of IVC related infections in the study included: type of cathether, greatest for nontunnelled lines [hazard ratio (HR): 3.50; $\mathrm{P}<0.0001$ ] and tunnelled lines (HR: 1.77; $\mathrm{P}<0.011$ ) compared to peripherally inserted central venous catheter (PICC) lines. Patients with tunneled catheters in our study were 1.4 times more likely to present with Staphylococcus aureus as compared to other types of catheters. Our results are consistent with previous studies done on patients with tunneled hemodialysis catheters which have shown gram-positive organisms to account for $63 \%$ of isolates, and $43 \%$ of the Gram-positive isolates were Staphylococcus aureus [20].

In conclusion, our study demonstrates an increase in Staphylococcus aureus indwelling venous catheter related infections. We further found a correlation between various organisms causing IVC related infections and various host and catheter related factors. These correlations impact the choice of initial presumptive antimicrobial therapy, duration of antimicrobial therapy and further management including removal of the source.

\section{Conflict of Interest}

All authors have read the journal's policy on conflicts of interest and have none to declare.

\section{References}

1. Centers for Disease Control and Prevention (CDC) (2011) Vital signs: central lineassociated blood stream infections-United States, 2001, 2008, and 2009. MMWR Morb Mortal Wkly Rep 60: 243-248.

2. Wisplinghoff H, Bischoff T, Tallent SM, Seifert H, Wenzel RP, et al. (2004) Nosocomial bloodstream infections in US hospitals: analysis of 24,179 cases from a prospective nationwide surveillance study. Clin Infect Dis 39: 309-317. [Crossref]

3. National Nosocomial Infections Surveillance (Nnis) System Report (2000) Data Summary from January 1992-June 2001, issued August 2001. American Journal Infection Control 29: 404-421

4. Mermel LA, Allon M, Bouza E (2009) Clinical practice guidelines for the diagnosis and management of intravascular catheter-related infection: 2009 Update by the Infectious Diseases Society of America. Clin Infect Dis 49: 1.

5. Viale P, Stefani S (2006) Vascular catheter-associated infections: a microbiological and therapeutic update. $J$ Chemother 18: 235-249.

6. Lee SC, Chen KS, Tsai CJ (2004) An outbreak of methicillin-resistant Staphylococcus aureus infections related to central venous catheters for hemodialysis. Infect Control Hosp Epidemiol 25: 678-684.

7. Nielsen J, Ladefoged S, Kolmos H (1998) Dialysis Catheter-Related Septicemia: Focus on Staphylococcus aureus Septicemia. Nephrol Dial Transplant 13: 2847-2852.

8. Berman DS, Schaefler S, Simberkoff MS (1987) Staphylococcus aureus Colonization in Intravenous Drug Abusers, Dialysis Patients and Diabetes. J Infect Dis 155: 829-831.

9. Saxena AK, Panhotra BR, Venkateshappa CK (2002) The impact of nasal carriage of methicillin-resistant and methicillin-susceptible Staphylococcus a ureus (MRSA \& MSSA) on vascular access-related septicemia among patients with type-II diabetes on dialysis. Ren Fail 24: 763-777.

10. von Eiff C, Becker K, Machka K, Stammer H, Peters G (2001) Nasal carriage as a source of Staphylococcus aureus bacteremia. Study Group. N Engl J Med 344: 11-16. [Crossref]

11. Grothe C, da Silva Belasco AG, de Cássia Bittencourt AR, Vianna LA, de Castro Cintra Sesso R, et al. (2010) Incidence of bloodstream infection among patients on hemodialysis by central venous catheter. Rev Lat Am Enfermagem 18: 73-80.

12. Allon M (2004) Dialysis catheter-related bacteremia: treatment and prophylaxis. Am J Kidney Dis 44: 779-791. [Crossref]

13. Jacobsson G, Dashti S, Wahlberg T, Andersson R (2007) The epidemiology of and risk factors for invasive Staphylococcus aureus infections in western Sweden. Scand J Infect Dis 39: 6.

14. Marr KA, Sexton DJ, Conlon PJ, Corey GR, Schwab SJ, et al. (1997) Catheterrelated bacteremia and outcome of attempted catheter salvage in patients undergoing hemodialysis. Ann Intern Med 127: 275. 
15. Tacconelli E, Tumbarello M, Pittiruti M (1997) Central venous catheter-related sepsis in a cohort of 366 hospitalised patients. Eur J Clin Microbiol Infect Dis 16: 203-209.

16. Armstrong CW, Mayhall CG, Miller KB (1986) Prospective study of catheter replacement and other risk factors for infection of hyperalimentation catheters. Journal of Infectious Diseases 154: 808-816.

17. Tumbarello M, Tacconelli E, Caponera S, Cauda R, Ortona L (1995) The impact of bacteraemia on HIV infection. Nine years experience in a large Italian university hospital. Journal of Infection 31: 123-131.
18. Swindell K, Lattif AA, Chandra J (2009) Parenteral lipid emulsion induces germination of Candida albicans and increases biofilm formation on medical catheter surfaces. $J$ Infect Dis 200: 473

19. Mollee P, Jones M, Stackelroth J (2011) Catheter-associated bloodstream infection incidence and risk factors in adults with cancer: a prospective cohort study. $J$ Hosp Infect 78: 26-30.

20. Mokrzycki MH, Zhang M, Cohen H, Golestaneh L, Laut JM, et al. (2006) Tunnelled haemodialysis catheter bacteraemia: risk factors for bacteraemia recurrence, infectious complications and mortality. Nephrol Dial Transplant 21: 1024-1031.

Copyright: $\odot 2018$ Shahani L. This is an open-access article distributed under the terms of the Creative Commons Attribution License, which permits unrestricted use, distribution, and reproduction in any medium, provided the original author and source are credited. 